\title{
Sistema computacional: Avaliação do impacto econômico da
} mastite

$\underline{\text { Marcos Aurélio Lopes }}{ }^{1}$; $\underline{\text { Glauber dos } \operatorname{Santos}^{2}}$; Geraldo Márcio da Costa ${ }^{3}$; Fabiana Alves

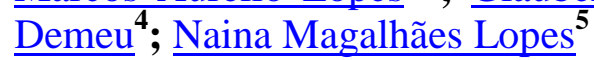

${ }^{1}$ Professor Titular do Departamento de Medicina Veterinária da Universidade Federal de Lavras, Bolsista do CNPq.

${ }^{2}$ Pós-graduando em Ciências Veterinárias na Universidade Federal de Lavras

${ }^{3}$ Professor do Departamento de Medicina Veterinária da Universidade Federal de Lavras

${ }^{4}$ Pós graduanda em Ciências Veterinárias na Universidade Federal de Lavras, professora do IFRO, bolsista pró doutoral

${ }^{5}$ Graduanda em Medicina Veterinária na Universidade Federal de Lavras

*Autor para correspondência, E-mail: malopes@dmv.ufla.br

RESUMO. Objetivou-se desenvolver um sistema computacional para auxiliar os técnicos e produtores a estimarem o impacto econômico da mamite em rebanhos leiteiros, desenvolver uma ferramenta que possibilite ao técnico e ou produtor efetuar simulações envolvendo diversos índices e parâmetros zootécnicos em um sistema de produção de leite e apresentar o sistema computacional CU\$TO MAMITE. Tal sistema foi desenvolvido utilizando planilha eletrônica, o Microsoft Excel. As rotinas foram desenvolvidas de forma conversacional, com acesso às diversas planilhas por meio de botões auto-explicativos. O CU\$TO MAMITE é uma importante ferramenta que auxilia o técnico e o pecuarista na estimativa do impacto da mamite, fornecendo informações importantes para a tomada de decisões pelos pecuaristas; e permite ao usuário diversas simulações englobando diversos parâmetros e índices envolvidos em um sistema de produção de leite.

Palavras chave: economia, informática, pecuária de leite, simulação.

\section{Computational system: evaluation of the mastitis economic impact}

\begin{abstract}
This work was intended to develop a computational system to aid both technicians and farmers to estimate the economic impact of mastitis in dairy herds; to develop a tool which enables the technician and or farmer to effect simulations involving a number of animal performance indices and parameters in a milk production system; and present the CU\$TO MAMITE computational system. Such a system was developed by utilizing the Microsoft Excel. Then routines were developed in a conversional way with access to several spreadsheets by means of self- explaining buttons. The CU\$TO MAMITE is an important tool which aids the technician and breeder in estimating the impact of mastitis, presenting important information for decision-making by the cattle raisers; and allow to the user several simulations encompassing a great deal of parameters and indices involved in a system of milk production.
\end{abstract}

Key words: Computer science, dairy production, profitability, simulation

\section{Introdução}

A mamite é a doença infecciosa mais comum do gado leiteiro e que mais causa prejuízos, incluindo a redução da produção e na qualidade do leite, o aumento dos custos com tratamentos e até mesmo o descarte precoce das vacas com mamite crônica (Müller, 2000). Durante as últimas décadas, o impacto econômico da mamite em fazendas leiteiras tem sido o motivo de várias pesquisas. Mesmo assim, continua sendo a doença que mais causa prejuízos à indústria leiteira, afetando diretamente o produtor, a indústria processadora e o consumidor final. De acordo com Santos (2002), no Brasil, em função 
da alta prevalência de mamite nos rebanhos, pode ocorrer perda de produção entre 12 e $15 \%$, o que significa um total de 2,8 bilhões de litros/ano em relação à produção anual de 21 bilhões de litros.

A mamite, quando na forma clínica, é de fácil diagnóstico e apresenta sinais clínicos evidentes; porém, o tipo que mais preocupa é a subclínica, pois é difícil de ser identificada e não apresenta sintomas visíveis, tornando-se responsável por aproximadamente $70 \%$ das perdas econômicas decorrentes de mamite (Müller, 2002).

De acordo com Fetrow et al. (2000), a análise de custos da mamite em rebanhos leiteiros deveria incluir todas as perdas associadas à ocorrência da doença, assim como os custos das medidas aplicadas no seu controle. No entanto, devido ao seu caráter multifatorial, algumas medidas de controle não podem ser identificadas exclusivamente como tendo o objetivo de controlar a mamite, como por exemplo: manutenção de ambiente limpo e seco, estratégias para aumento do consumo de alimentos, melhorias no conforto dos animais e eficiência no tempo de ordenha, o que dificulta a quantificação desses custos com relação à mamite.

Santos (2002) salientou que o custo da mamite pode variar em função do rebanho e do tempo. Diversos fatores podem afetar o custo final da mamite, uma vez que esses itens podem sofrer variações ao longo do tempo: preço do leite, custos de alimentação, custos de reposição de descartes e custos de medicamentos. A consideração desses fatores em cada situação permite uma definição mais precisa dos custos da mamite em um rebanho em particular.

O crescimento da competição, cada vez mais acirrada, evidencia a necessidade que empresários do setor têm de tomar decisões acertadas, ou seja, uma decisão baseada apenas na intuição ou no conhecimento empírico da situação pode significar, em curto prazo, prejuízo e em longo prazo até mesmo a exclusão da empresa do mercado. Sabe-se que a informática é uma importante ferramenta que pode auxiliar os empresários e técnicos no processo de tomada de decisão. No processo de informatização e modernização da pecuária, diversas são as aplicações e usos da informática, muitas dessas relatadas por Lopes (1997) e Lopes, (2002). Dentre essas, destaca-se o desenvolvimento de sistemas computacionais, visando ajudar pecuaristas e profissionais a tomarem uma decisão correta.
Considerando a alta demanda de sistemas computacionais destinados a estimar o impacto da mamite na rentabilidade de pecuaristas de leite, a complexidade de tal tarefa e a escassez de ferramentas computacionais, este trabalho teve como objetivos: 1) desenvolver um sistema computacional para auxiliar os técnicos e produtores a estimarem o impacto econômico da mamite em rebanhos leiteiros; 2) desenvolver uma ferramenta que possibilite ao técnico e ou produtor efetuar simulações envolvendo diversos índices e parâmetros zootécnicos em um sistema de produção de leite; e 3) apresentar o sistema computacional CU\$TO MAMITE, cuja finalidade é estimar o impacto econômico da mamite em um sistema de produção de leite.

\section{Material e Métodos}

O Sistema computacional foi desenvolvido utilizando planilha eletrônica, o Microsoft Excel. O impacto da mamite (IM) foi estimado levandose em consideração todas as despesas operacionais efetivas (Lopes \& Carvalho, 2000), sendo:

- IM= Total em perdas + medidas preventivas + tratamento curativo, onde:

- Total de perdas $=$ perdas em leite (redução na produção e descarte de leite durante o tratamento e período de carência do antibiótico);

- Medidas preventivas $=$ despesas com antibiograma e cultura + despesas com realização de exame de contagem de células somáticas no tanque (CCST) + exames de contagem de células somáticas individuais (CCSI) + pré-dipping + pós-dipping + tratamento de vacas secas + vacinação de vacas secas + manutenção de ordenhadeira;

- Tratamento curativo $=$ despesas com aplicação do antibiótico local + antibiótico sistêmico + antiinflamatório.

Para estimar o custo da mão de obra, considerou-se o tempo gasto (em minutos) para realização da atividade relacionada com mastite, multiplicado pelo quanto custava o minuto do funcionário para a fazenda. Tais atividades foram: aplicação de medicamentos intramamários, coleta de leite para análises laboratoriais, pré e pósdipping, vacinação. Os tempos de $10 ; 5 ; 1 ; 0,5$ e 5 minutos respectivamente, foram obtidos por meio da média de três funcionários de uma fazenda no município de Lavras / MG. Considerou-se um salário de R \$788,00/mês, ou 
seja, por minuto, esse funcionário custava $\mathrm{R} \$$ 0,05 . Os preços dos produtos e exames utilizados foram os praticados na região de Lavras, no mês de janeiro de 2015.

As perdas por redução na produção de leite devido à mastite foram estimadas utilizando a equação de regressão linear, obtida a partir dos dados do NMC (1996):

$-\mathrm{y}=-4,7908+0,0226 \mathrm{x}$.

Na qual "y" é a porcentagem de perda de leite e " $x$ " a contagem de células somáticas.

$O$ percentual de quartos acometidos pela mastite subclínica foi estabelecido em 9,36\% através da equação de regressão linear obtida junto ao NMC (1996):

- y $=-0,2959+0,0322 x$, em que: y é o percentual de quartos afetados pela mastite subclínica; $x$ é a quantidade de células somáticas no tanque (CCST x 1000 células/mL).

$O$ custo com a reposição de matrizes descartadas por mastite foi obtido pelo valor de aquisição de uma nova matriz subtraído o valor de venda da vaca vendida para o abate.

\section{Resultados e Discussão}

As rotinas foram desenvolvidas de forma conversacional, com acesso às diversas planilhas por meio de botões auto-explicativos, o que permite a operação do sistema por pessoas não especializadas em informática, mas apenas treinadas na operação de microcomputadores. Tal fato possibilita um maior uso do sistema computacional no meio rural, onde a informática não é muito difundida (Lopes, 1997).

A tela de abertura possui botões para auxiliarem na navegação entre as 14 planilhas pertencentes aos grupos parâmetros (Cadastro, Índices, Perdas e Preços), medidas curativas (Casos Clínicos), medidas preventivas (Monitoramento, Pré e Pós-dipping, Vacinação e Vacas secas), impacto econômico (Resultado) e planilhas auxiliares (Gráfico de perdas, Mão de obra e Help) (Figura 1).

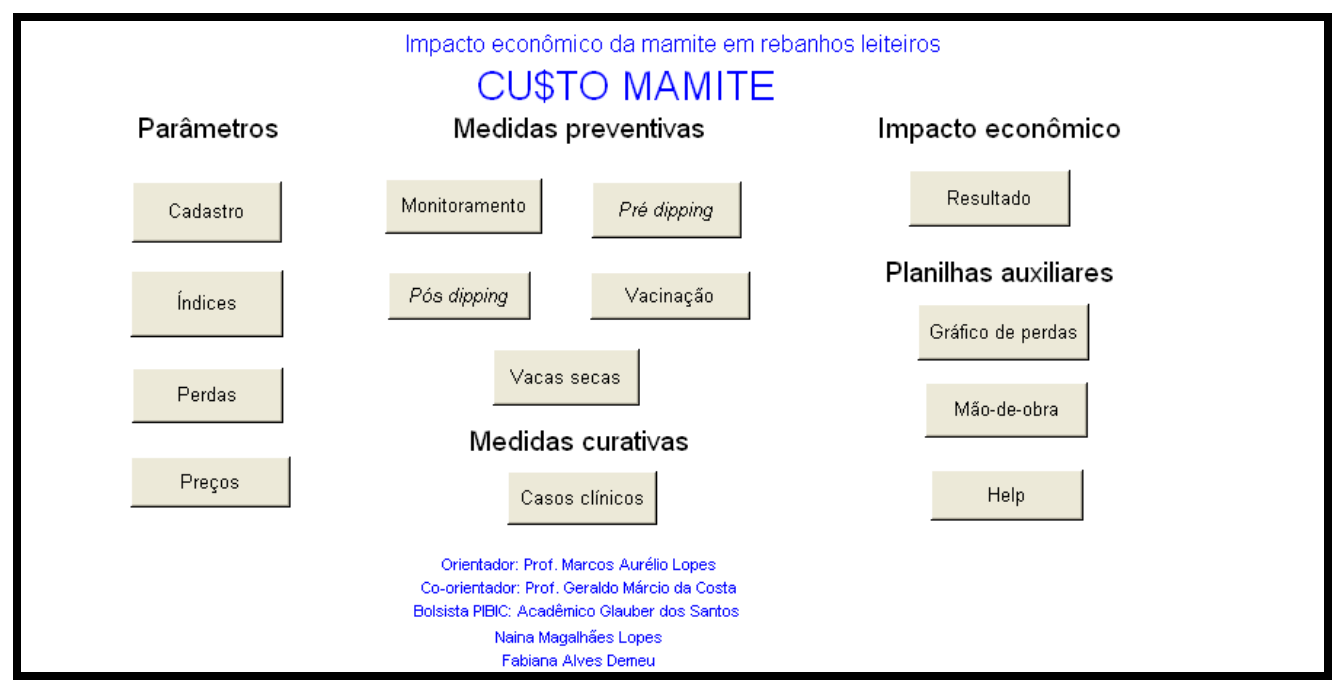

Figura 1. Tela de abertura do CU\$TO MAMITE

Para selecionar uma opção o usuário deve posicionar o mouse e clicar sobre a opção desejada. Esse recurso facilita a operação do sistema computacional, mostrando ao usuário todas as opções disponíveis. Em todas as planilhas, exceto na "Abertura", no canto superior esquerdo há um botão Abertura, que permite o retorno à tela de abertura. Clicando sobre ele, a planilha contendo o mеnи de abertura será mostrada na tela do monitor, possibilitando, assim, uma navegação mais rápida no sistema computacional.

Para estimar o impacto econômico da mamite em rebanhos leiteiros o usuário, em Cadastros, deve lançar os dados referentes à propriedade em estudo, que, no exemplo hipotético em questão estão apresentados na Tabela 1 
Tabela 1. Tela contendo a ficha de cadastro dos dados de um sistema de produção de leite hipotético utilizados na simulação

\begin{tabular}{lr}
\hline & Cadastro \\
\hline Quantidade de vacas em lactação & 100 \\
$\%$ de vacas secas & 33 \\
Produção diária \animal (kg leite) & 20 \\
CCS (Contagem de Células Somáticas) & 500.000 \\
Frequência de ordenhas \dia & 2 \\
IEP (Intervalo de parto) (meses) & 15 \\
Percentual de casos clínicos \mês & 7 \\
Quantidade de quartos com mamite & 1 \\
Valor do custo do leite \kg & 3 \\
Quantidade de doses de vacina \vaca seca & 20 \\
$\%$ de descarte devido mamite & 1
\end{tabular}

$\begin{array}{lr}\text { Valor de aquisição da vaca } & \mathrm{R} \$ 6.750,00\end{array}$

$\begin{array}{lr}\text { Valor de venda da vaca a ser descartada } & \mathrm{R} \$ 2.214,00\end{array}$

Tratamento utilizado

Antibiótico

Antibiótico intramamário

Antibiótico sistêmico

Em índices, estão alguns dados que devem ser preenchidos pelo usuário e ainda outros calculados pelo sistema computacional, permitindo que o usuário simule várias situações possíveis, em função das mudanças que podem ocorrer no sistema de produção ao longo do ano. A planilha Perdas possui as informações calculadas sobre o quanto de leite a vaca deixou de produzir em função da quantidade da CCS (em $\mathrm{kg} \mathrm{e} \mathrm{\% ),} \mathrm{além} \mathrm{da} \mathrm{\%} \mathrm{e} \mathrm{quantidade} \mathrm{de} \mathrm{quartos}$ infectados, descarte de leite residual, descarte de vacas e morte em decorrência da mamite. O usuário deverá ainda "alimentar" a planilha Preços, cadastrando todos os valores referentes aos produtos e serviços utilizados nas medidas preventivas e curativas da mamite. Lançados esses valores, o sistema estima o impacto econômico da mamite.

Ao clicar em "Monitoramento", o usuário irá se deparar com todas as despesas de monitoramento da mamite, dividida em itens de despesas tais como: antibiograma, cultura, CCST
(Contagem de células somáticas do tanque), CMT (Califórnia mastit test) e CCSI (Contagem de células somáticas individuais). Nessas, existem os subitens: custo do antibiograma (laboratório), mão de obra, tubo para coleta de leite, isopor, sedex; custo da cultura (laboratório), mão de obra e caneca para coleta de leite, isopor, sedex e CCST: valor da bandeja, mão de obra e reagente; CCSI: valor da bandeja, mão de obra e reagente. Há ainda colunas contendo o valor total, a porcentagem de cada subitem dentro de cada despesa e a porcentagem desta dentro do grupo monitoramento. Esse último mostra ao empresário qual a porcentagem que um determinado item representa dentro de um determinado grupos de despesas (Figura 2).

Nas planilhas pré (Figura 3) e pós dipping (Figura 4) podem ser visualizadas as despesas com aquisição de materiais e produtos necessários na realização dessas práticas. Vacinações visando à prevenção de mamite durante o período seco (de descanso), bem como 
as despesas decorrentes como agulha, seringas etc, poderão ser visualizadas na planilha vacinação (Figura 5). Os itens que compõe a manutenção de ordenhadeira, bem como suas respectivas quantidades e preços podem ser visualizados na planilha manutenção de ordenhadeira (Figura 6).

\begin{tabular}{|c|c|c|c|c|c|c|c|c|}
\hline \multicolumn{9}{|c|}{ Despesas com monitoramento } \\
\hline \multirow{3}{*}{\begin{tabular}{l}
\multicolumn{1}{c}{ Despesas } \\
Antiograma e Cultura \\
Custo do antibiograma (laboratório)
\end{tabular}} & \multirow{3}{*}{$\begin{array}{l}\text { Unidade } \\
\text { und }\end{array}$} & \multicolumn{2}{|c|}{ Valor (R\$) } & \multirow{2}{*}{\multicolumn{2}{|c|}{$\begin{array}{c}\text { Quantidade } \\
\text { Anual }\end{array}$}} & \multirow{3}{*}{$\begin{array}{l}\text { Gasto } \\
\text { Anual } \\
\qquad 378,00\end{array}$} & \multirow{3}{*}{$\begin{array}{c}\% \\
\text { Itibiograr } \\
\% \\
18,5 \%\end{array}$} & \multirow{3}{*}{$\begin{array}{c}\text { monitoramento } \\
5,8 \%\end{array}$} \\
\hline & & Unit & tário & & & & & \\
\hline & & $\mathrm{R} \$$ & 9,00 & 42 & $\mathrm{R} \$$ & & & \\
\hline Custo da cultura (laboratório) & und & $\mathrm{R} \$$ & 10,00 & 46 & $\mathrm{R} \$$ & 460,00 & $22,6 \%$ & $7,0 \%$ \\
\hline Caixa de isopor & und & $\mathrm{R} \$$ & 4,90 & 2 & $\mathrm{R} \$$ & 9,80 & $0,5 \%$ & $0,2 \%$ \\
\hline Recipiente para envio de leite ao laboratório & und & $\mathrm{R} \$$ & 0,80 & 2 & $\mathrm{R} \$$ & 1,60 & $0,1 \%$ & $0,0 \%$ \\
\hline Sedex & und & $\mathrm{R} \$$ & 65,00 & 2 & $\mathrm{R} \$$ & 130,00 & $6,4 \%$ & $2,0 \%$ \\
\hline Mão-de-obra para coleta do leite & $\mathrm{R} \$ /$ dia & $\mathrm{R} \$$ & 520,86 & 2 & $\mathrm{R} \$$ & $1.041,72$ & $51,1 \%$ & $15,9 \%$ \\
\hline Caneca para coleta de leite & und & $\mathrm{R} \$$ & 18,00 & 1 & $\mathrm{R} \$$ & 18,00 & $0,9 \%$ & $0,3 \%$ \\
\hline \multirow[t]{2}{*}{ Sub-Total antibiograma e cultura } & & & & & R\$ & $2.039,12$ & $100,0 \%$ & $31,2 \%$ \\
\hline & & & & & & & $\%$ & \\
\hline \multicolumn{2}{|c|}{ CCST (Contagem de células somática do tanque) } & & & & & & CCST & monitoramento \\
\hline Laboratório & und & $\mathrm{R} \$$ & 1,30 & 12 & $\mathrm{R} \$$ & 15,60 & $1,9 \%$ & $0,2 \%$ \\
\hline Recipiente para envio de leite ao laboratório & und & $\mathrm{R} \$$ & 0,80 & 2 & $\mathrm{R} \$$ & 1,60 & $0,2 \%$ & $0,0 \%$ \\
\hline Sedex & und & $\mathrm{R} \$$ & 65,00 & 12 & $\mathrm{R} \$$ & 780,00 & $96,3 \%$ & $11,9 \%$ \\
\hline Caixa de isopor & und & $\mathrm{R} \$$ & 4,90 & 2 & $\mathrm{R} \$$ & 9,80 & $1,2 \%$ & $0,2 \%$ \\
\hline Mão-de-obra para coleta do leite & $\mathrm{R} \$ / \mathrm{min}$ & $\mathrm{R} \$$ & 0,05 & 60 & $\mathrm{R} \$$ & 3,24 & $0,4 \%$ & $0,0 \%$ \\
\hline \multirow[t]{2}{*}{ Sub-Total CCST } & & & & & $\mathbf{R} \$$ & 810,24 & $100,0 \%$ & $12,4 \%$ \\
\hline & & & & & & & $\%$ & \\
\hline \multirow{5}{*}{$\begin{array}{l}\text { CMT (Califormia Mastit Test) } \\
\text { Reagente } \\
\text { Mão-de-obra especializada } \\
\text { Valor da bandeja } \\
\text { Sub-total CMT }\end{array}$} & & & & & & & СMT & monitoramento \\
\hline & $\mathrm{ml}$ & $\mathrm{R} \$$ & 0,02 & 0 & $\mathrm{R} \$$ & - & \#DIV/0! & $0,0 \%$ \\
\hline & $\mathrm{R} \$ / \mathrm{min}$ & $\mathrm{R} \$$ & 1,09 & 0 & $\mathrm{R} \$$ & - & \#DIV/0! & $0,0 \%$ \\
\hline & und & $\mathrm{R} \$$ & 17,70 & 0 & $\mathrm{R} \$$ & - & \#DIV/0! & $0,0 \%$ \\
\hline & & & & & $\mathbf{R} \$$ & - & \#DIV/0! & $0,0 \%$ \\
\hline \multirow{2}{*}{\multicolumn{2}{|c|}{ CCSI (Contagem de células somáticas individual) }} & & & & & & $\%$ & \\
\hline & & & & & & & $\operatorname{ccsl}$ & monitoramento \\
\hline Laboratório & und & $\mathrm{R} \$$ & 1,30 & 1.200 & $\mathrm{R} \$$ & $1.560,00$ & $42,4 \%$ & $23,9 \%$ \\
\hline Mão-de-obra para coleta do leite & $\mathrm{R} \$ / \mathrm{min}$ & $\mathrm{R} \$$ & $0,05^{\prime}$ & 6.000 & $\mathrm{R} \$$ & 323,84 & $8,8 \%$ & $5,0 \%$ \\
\hline Recipiente para envio de leite ao laboratório & und & $\mathrm{R} \$$ & 0,80 & 1.200 & $\mathrm{R} \$$ & 960,00 & & \\
\hline Caixa de isopor & und & $\mathrm{R} \$$ & 4,90 & 12 & $\mathrm{R} \$$ & 58,80 & $2,9 \%$ & $0,9 \%$ \\
\hline Sedex & und & $\mathrm{R} \$$ & 65,00 & 12 & $\mathrm{R} \$$ & 780,00 & $38,3 \%$ & $11,9 \%$ \\
\hline Sub-total CCSI & & & & & R\$ & $3.682,64$ & $100,0 \%$ & $56,4 \%$ \\
\hline Total de despesas com monitoramento & & & & & $\mathrm{R} \$$ & $6.531,99$ & & $100,0 \%$ \\
\hline
\end{tabular}

Figura 2. Tela contendo a planilha despesas com monitoramento

\begin{tabular}{|c|c|c|c|c|c|c|c|}
\hline \multicolumn{8}{|c|}{ Despesas com pré-dipping } \\
\hline Despesas & Unidade & $\begin{array}{l}\text { Val } \\
\text { Un }\end{array}$ & $\begin{array}{l}\text { r (R\$) } \\
\text { itario }\end{array}$ & $\begin{array}{c}\text { Quantidade } \\
\text { Anual }\end{array}$ & & $\begin{array}{l}\text { Gasto } \\
\text { Anual }\end{array}$ & $\%$ \\
\hline Solução pré dipping & $\mathrm{ml}$ & RS & 0,004 & 365.000 & R\$ & $1.587,75$ & $94,6 \%$ \\
\hline Papel toalha & und & R\$ & 0,005 & 5.840 & R\$ & 28,62 & $1,7 \%$ \\
\hline Mão-de-obra & $\mathrm{R} \$ / \min$ & R\$ & 0,054 & 730 & R\$ & 39,40 & $2,3 \%$ \\
\hline Valor do copo para pré dipping & und & R\$ & 22,00 & 1 & $\mathrm{R} \$$ & 22,00 & $1,3 \%$ \\
\hline Total & & & & & $\mathrm{R} \$$ & $1.677,77$ & $100,0 \%$ \\
\hline
\end{tabular}

Figura 3. Tela contendo a planilha despesas com pré - dipping

\begin{tabular}{|c|c|c|c|c|c|c|}
\hline \multicolumn{7}{|c|}{ Despesas com Pós-dipping } \\
\hline Despesas & Unidade & $\begin{array}{c}\text { Valor (R\$) } \\
\text { Unitario }\end{array}$ & $\begin{array}{c}\text { Quantidade } \\
\text { Anual }\end{array}$ & & $\begin{array}{l}\text { Gasto } \\
\text { Anual }\end{array}$ & $\%$ \\
\hline Solução de pós dipping & $\mathrm{ml}$ & $\mathrm{R} \$ 0,0087$ & 365.000 & $\mathrm{R} \$$ & $3.175,50$ & $98,7 \%$ \\
\hline Mão-de-obra & $\mathrm{R} \$ / \mathrm{min}$ & $\mathrm{R} \$ 0,0540$ & 365 & $\mathrm{R} \$$ & 19,70 & $0,6 \%$ \\
\hline Valor do copo para pós dipping & und & $\mathrm{R} \$ 22,00$ & 1 & $\mathrm{R} \$$ & 22,00 & $0,7 \%$ \\
\hline Total & & & & $\mathrm{R} \$$ & $3.217,20$ & $100,0 \%$ \\
\hline
\end{tabular}

Figura 4. Tela contendo a planilha despesas com pós - dipping 


\begin{tabular}{|c|c|c|c|c|c|}
\hline \multicolumn{6}{|c|}{ Despesas com vacinação } \\
\hline & & Valor (R\$) & Quantidade & Gasto & $\%$ \\
\hline Despesas & Unidade & Unitario & Anual & Anual & \\
\hline Agulha & und & $\mathrm{R} \$ 1,50$ & 320 & $\mathrm{R} \$ 479,99$ & $23,7 \%$ \\
\hline Seringa & und & $\mathrm{R} \$ 0,80$ & 320 & $\mathrm{R} \$ 255,99$ & $12,7 \%$ \\
\hline Vacina & und & $\mathrm{R} \$ 3,75$ & 320 & R\$ $1.199,97$ & $59,3 \%$ \\
\hline Mão-de-obra permanente & $\mathrm{R} \$ / \mathrm{min}$ & $\mathrm{R} \$ 0,05$ & 1.600 & $\mathrm{R} \$ 86,35$ & $4,3 \%$ \\
\hline Total & & $\mathrm{R} \$ 6,10$ & & $\mathrm{R} \$ 2.022,31$ & $100,0 \%$ \\
\hline
\end{tabular}

Figura 5. Tela contendo a planilha despesas com vacinação

\begin{tabular}{|c|c|c|c|c|}
\hline \multicolumn{5}{|c|}{ Manutenção de Ordenhadeiras } \\
\hline & & Valor (R\$) & Quantidade & Gasto \\
\hline Despesas & Unidade & Unitario & Anual & Anual \\
\hline Mangueiras (tubo longo duplo vácuo) & Und & $\mathrm{R} \$ 29,60$ & 12 & $\mathrm{R} \$ 355,20$ \\
\hline Mangueiras (tubo longo vácuo) & Und & $\mathrm{R} \$ 18,40$ & 12 & $\mathrm{R} \$ 220,80$ \\
\hline Isulfladores (teteiras) & Conj & $\mathrm{R} \$ 48,00$ & 1,75 & $\mathrm{R} \$ 84,10$ \\
\hline Oleo da bomba de vácuo & Lt & $\mathrm{R} \$ 11,40$ & 75,92 & $\mathrm{R} \$ 865,49$ \\
\hline Manutenção ordenhadeira & Und & $\mathrm{R} \$ 250,00$ & 2 & $\mathrm{R} \$ 500,00$ \\
\hline Total & & & & $\mathrm{R} \$ 2.025,58$ \\
\hline
\end{tabular}

Figura 6. Tela contendo a planilha manutenção de ordenhadeira

As despesas com tratamento preventivo em vacas secas e tratamento de casos clínicos de vacas acometidas de mamite estão relacionadas nas planilhas vacas secas (Figura 7) e casos clínicos (Figura 8), respectivamente. O sistema computacional permite uma visualização detalhada, com relação a todas as despesas relacionadas com a infecção da glândula mamária, bem como suas porcentagens no controle da doença.

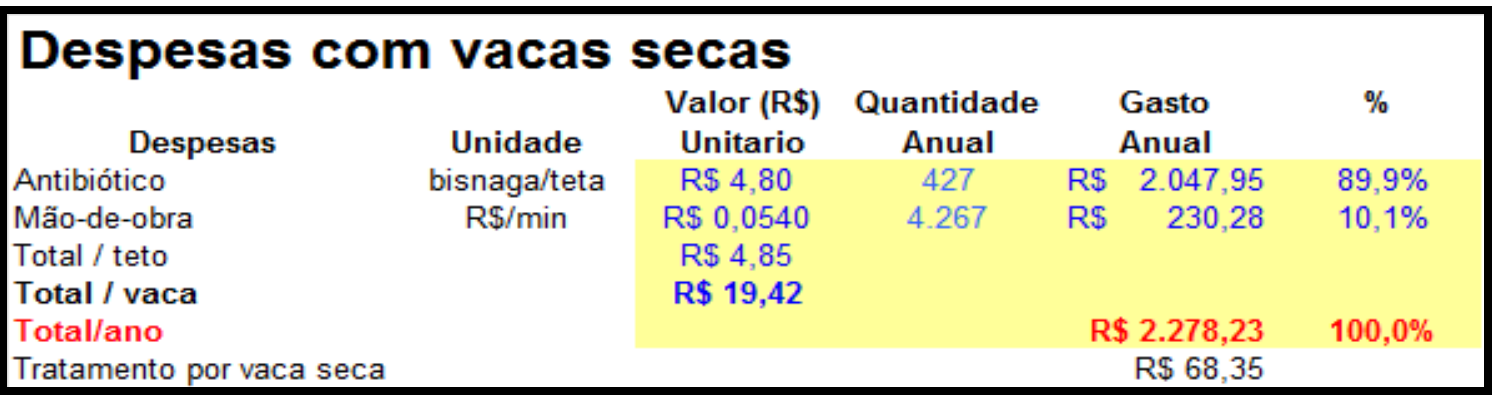

Figura 7. Tela contendo a planilha despesas com vacas secas

Um resumo do impacto econômico da mamite em um sistema de produção de leite pode ser visualizado na planilha Relatório (Figura 9), no qual estão estimados o custo do tratamento preventivo ( $R \$ 0,0237)$ e curativo ( $R \$ 0,0605)$, bem como o impacto econômico da mamite $(\mathrm{R} \$$ 0,3961), por kg de leite. Diante desse relatório, é possível visualizar com maior clareza quais são as principais despesas, perdas, e o mais importante, onde não se deve preocupar em reduzir gastos, como por exemplo, na prevenção, pois tal item apresenta baixo custo e mostra-se altamente benéfico. Ribeiro et al. (2006) verificaram que a relação custo/benefício foi satisfatória, uma vez que, para cada $R \$ 1,00$ de investimento houve de $\mathrm{R} \$ 2,90$ a $\mathrm{R} \$ 5,30$ de renda adicional. O relatório traz ainda o custo das medidas preventivas e curativas por vaca tratada, bem como um resumo das despesas com prevenção, tratamento curativo e das perdas provocadas pela mamite no sistema de produção hipotético utilizado na simulação. 


\begin{tabular}{|c|c|c|c|c|c|c|c|}
\hline \multicolumn{8}{|c|}{ Despesas com casos clínicos } \\
\hline \multicolumn{2}{|c|}{ Despesas } & \multirow{2}{*}{\multicolumn{2}{|c|}{$\begin{array}{c}\text { valor (R\$) / } \\
\text { dia }\end{array}$}} & \multirow{2}{*}{\multicolumn{2}{|c|}{$\begin{array}{l}\text { Gasto } \\
\text { anual }\end{array}$}} & \multirow{5}{*}{$\begin{array}{c}\% \\
\text { Trat local } \\
96,9 \% \\
3,1 \% \\
100,0 \%\end{array}$} & \multirow{5}{*}{$\begin{array}{c}\% \\
\text { Trat.sist } \\
57,1 \% \\
1,8 \% \\
58,9 \%\end{array}$} \\
\hline Tratamento local & unidade & & & & & & \\
\hline Antibiótico local & $\mathrm{ml}$ & $\mathrm{R} \$$ & 82,35 & $\mathrm{R} \$$ & $30.058,82$ & & \\
\hline Mão-de-obra & $\mathrm{R} \$ / \mathrm{dia}$ & $\mathrm{RS}$ & 2,67 & $\mathrm{R} \$$ & 973,41 & & \\
\hline Sub -Total local & & $\mathrm{RS}$ & 85,02 & $\mathrm{R} \$$ & $31.032,24$ & & \\
\hline Tratamento local / vaca / dia de tratamento & & R\$ & 17,21 & & & & \\
\hline Total / vaca / tratamento local & & $\mathbf{R} \$$ & 51,62 & & & & \\
\hline Total tratamento local / vaca em lactação & & $\mathbf{R} \$$ & 310,32 & & & & \\
\hline Tratamento sistêmico & & & & & & rat. sistêmi & \\
\hline Agulha (RS / dia) & und & R\$ & 1.50 & RS & 1.127 .21 & $5,2 \%$ & $2,1 \%$ \\
\hline Anti-inflamatório sistêmico ( $\mathrm{R} \$ / \mathrm{dia} /$ vacas trat) & & R\$ & 8,91 & $\mathrm{R} \$$ & $1.339,12$ & $6,2 \%$ & $2,5 \%$ \\
\hline Seringa & und & $\mathrm{R} \$$ & 1,65 & $\mathrm{R} \$$ & 601,18 & $2,8 \%$ & $1,1 \%$ \\
\hline Mão-de-obra anti inflamatorio local & $\mathrm{R} \$ / \mathrm{dia}$ & $\mathrm{R} \$$ & 1,11 & $\mathrm{R} \$$ & 405,59 & $1,9 \%$ & $0,8 \%$ \\
\hline Anti inflamatório local & $\mathrm{ml}$ & $\mathrm{R} \$$ & 3.67 & $\mathrm{R} \$$ & $1.339,12$ & $6,2 \%$ & $2,5 \%$ \\
\hline Agulha ( $\mathrm{R} \$ /$ dia $)$ & und & $\mathrm{R} \$$ & 3,09 & R\$ & $1.127,21$ & $5,2 \%$ & $2,1 \%$ \\
\hline Antibiótico sistêmico ( $\mathrm{R} \$ / \mathrm{dia} /$ vacas trat) & & $\mathrm{R} \$$ & 19,04 & $\mathrm{R} \$$ & $6.951,10$ & $32,2 \%$ & $13,2 \%$ \\
\hline Seringa & und & $\mathrm{R} \$$ & 1,65 & $\mathrm{R} \$$ & 601,18 & $2,8 \%$ & $1,1 \%$ \\
\hline Mão-de-obra & $\mathrm{R} \$ / \mathrm{dia}$ & $\mathrm{RS}$ & 1,11 & $\mathrm{R} \$$ & 405,59 & $1,9 \%$ & $0,8 \%$ \\
\hline Antibiótico local & $\mathrm{ml}$ & $\mathrm{R} \$$ & 34,31 & R\$ & 12.524 .51 & $58,0 \%$ & $23.8 \%$ \\
\hline Tratamento sistêmico / vaca /dia & & $\mathbf{R} \$$ & 28,76 & $\mathbf{R} \mathbf{S}$ & $21.609,58$ & $100,0 \%$ & \\
\hline Total caso clínico (local + sistêmico) / dia & & $\mathbf{R} \$$ & 80,38 & $\mathbf{R} \$$ & $52.641,82$ & & \\
\hline Total / vaca / tratamento sistêmico & & $\mathbf{R} \$$ & 143,78 & & & & \\
\hline Tratamento sistemico / vaca em lactação & & $\mathbf{R} \$$ & 216,10 & & & & \\
\hline Número de casos clínicos/ano & & $\mathrm{R} \$$ & 751 & & & & \\
\hline Numero de casos brandos & & $\mathrm{R} \$$ & 601 & & $31.032,24$ & & \\
\hline Número de casos severos & & $\mathrm{R} \$$ & 150 & & $21.609,58$ & & \\
\hline Total tratamento (R\$) / ano (casos brandos + c & asos severo & & & & $52.641,82$ & & \\
\hline Total de tratamento $(\mathrm{R} \$$ ) (casos brandos + c & severo & & e vaca & & & & \\
\hline Total de tratamento (R\$) (casos brandos $+\mathrm{c}$ & severos) & & & & 254,72 & & \\
\hline
\end{tabular}

Figura 8. Tela contendo a planilha despesas com casos clínicos

\section{Relatório: impacto econômico da mamite}

Dados do sistema de produção de leite

Quantidade de vacas em lactação

CCS (Contagem de Células Somáticas) $(\times 1.000 / \mathrm{ml})$

Especificação

Custo da prevenção / kg de leite

Custo do tratamento curativo $/ \mathrm{kg}$ de leite

Custo do tratamento prev + curativo / $\mathrm{kg}$ de leite

Perdas / $\mathrm{kg}$ de leite

Impacto econômico / kg de leite

Custo do tratamento curativo de caso brando / vaca

Custo do tratamento curativo de caso severo / vaca

Custo da prevenção / vaca em lactação

Custo da prevenção/rebanho (vacas lactação + vacas secas)

Custo do tratamento curativo / vaca em lactação / ano

Custo do tratamento prev + curativo / vaca em lactação / ano

Perdas de leite (redução na produção+ descarte) / vaca

Impacto econômico / vaca em lactação / ano

Impacto econômico (perdas + despesas) / ano

Despesas com prevenção

Antibiograma e cultura

CCST (Contagem de Células Somáticas no tanque)

CCSI (Contagem de células somáticas individual)

Pós-dipping

Pré-dipping

ratamento de vaca seca

ratameto por vaca seca

Vacinação

Manutenção de ordenhadeira

Sub-total (preventivo)

Despesas com tratamento curativo

Antibiótico uso local

Antibiótico sistêmico

Sub-total (curativo)

Total (preventivo + curativo)

Perdas

Volume de leite perdido (redução na produção + descarte) ( $\mathrm{kg} / \mathrm{ano}$ )

Redução na produção / R\$ por ano

Descarte de leite residual / R\$ por ano

Perda em leite (redução na produção + descarte) $\mathrm{R} \$ / a n$

Desvalorização de matrizes

Morte de matrizes

Total de perdas (ano)

Impacto econômico (perdas + despesas) / ano

\begin{tabular}{|c|c|c|c|c|}
\hline & 100 & & & \\
\hline & 500 & & & \\
\hline & lor (R\$) & $\%$ & $\%$ & \\
\hline $\mathrm{R} \$$ & 0,0237 & $6,0 \%$ & & \\
\hline $\mathrm{R} \$$ & 0,0605 & $15,3 \%$ & & \\
\hline $\mathrm{R} \$$ & 0,0841 & & $21,2 \%$ & \\
\hline $\mathrm{R} \$$ & 0,3119 & $78,8 \%$ & $78,8 \%$ & \\
\hline $\mathrm{R} \$$ & 0,3961 & $100,0 \%$ & $100,0 \%$ & \\
\hline $\mathrm{R} \$$ & 51,62 & & & \\
\hline $\mathrm{R} \$$ & 143,78 & & & \\
\hline $\mathrm{R} \$$ & 206,02 & $28,1 \%$ & $6,0 \%$ & \\
\hline $\mathrm{R} \$$ & 154,52 & & & \\
\hline $\mathrm{R} \$$ & 526,42 & $71,9 \%$ & $15,3 \%$ & \\
\hline $\mathrm{R} \$$ & 732,44 & $100,0 \%$ & & \\
\hline $\mathrm{R} \$$ & $2.715,16$ & & $78,8 \%$ & \\
\hline $\mathrm{RS}$ & $3.447,60$ & & $100,0 \%$ & \\
\hline $\mathrm{R} \$ 3$ & 760,45 & & & \\
\hline & /ano (R\$) & $\%$ preventivo & $\%$ despesas & \\
\hline $\mathrm{R} \$$ & $2.039,12$ & $9,9 \%$ & $2,8 \%$ & \\
\hline $\mathrm{R} \$$ & 810,24 & $3,9 \%$ & $1,1 \%$ & \\
\hline R\$ & $6.531,99$ & $31,7 \%$ & $8,9 \%$ & \\
\hline $\mathrm{R} \$$ & $3.217,20$ & $15,6 \%$ & $4,4 \%$ & \\
\hline $\mathrm{R} \$$ & $1.677,77$ & $8,1 \%$ & $2,3 \%$ & \\
\hline $\mathrm{R} \$$ & $2.278,23$ & $11,1 \%$ & $3,1 \%$ & \\
\hline R\$ & 68,35 & & & \\
\hline $\mathrm{R} \$$ & $2.022,31$ & $9,8 \%$ & $2,8 \%$ & \\
\hline R\$ & $2.025,58$ & $9,8 \%$ & $2,8 \%$ & \\
\hline & $20.602,43$ & $100,0 \%$ & $28,1 \%$ & $5,98 \%$ \\
\hline & /ano (R\$) & $\%$ curativo & $\%$ despesas & \\
\hline $\mathrm{R} \$$ & $31.032,24$ & $58,9 \%$ & $42,4 \%$ & \\
\hline $\mathrm{R} \$$ & $21.609,58$ & $41,1 \%$ & $29,5 \%$ & \\
\hline R\$ & $52.641,82$ & $100,0 \%$ & $71,9 \%$ & $15,27 \%$ \\
\hline R\$ & $73.244,25$ & & $100,0 \%$ & \\
\hline & $.575,74$ & & & \\
\hline & $5.238,70$ & & $20,3 \%$ & $16,02 \%$ \\
\hline & $18.807,50$ & & $43,8 \%$ & $34,46 \%$ \\
\hline R\$ & $174.046,20$ & & $64,1 \%$ & $50,48 \%$ \\
\hline $\mathrm{R} \$$ & $90.720,00$ & & $33,4 \%$ & $26,31 \%$ \\
\hline RS & $6.750,00$ & & $2,5 \%$ & $1,96 \%$ \\
\hline R\$ & $271.516,20$ & & $100,0 \%$ & \\
\hline $\mathrm{R} \$$ & $344.760,45$ & & & \\
\hline
\end{tabular}

Figura 9. Tela contendo o Relatório do impacto econômico da mamite 
Constituem o grupo planilha auxiliar aquela nas quais há o Gráfico de Perdas (Figura 10), informações detalhadas sobre Mão de obra (Figura 11) e o Help, onde estão as equações de predição de perdas no leite em virtude da mamite, o custo temporal da mão de obra, ou seja, quanto custa o minuto de serviço das pessoas envolvidas com atividades desenvolvidas na prevenção e controle da mamite e uma ajuda, mostrando como se deve trabalhar com o CU\$TO MAMITE, respectivamente.
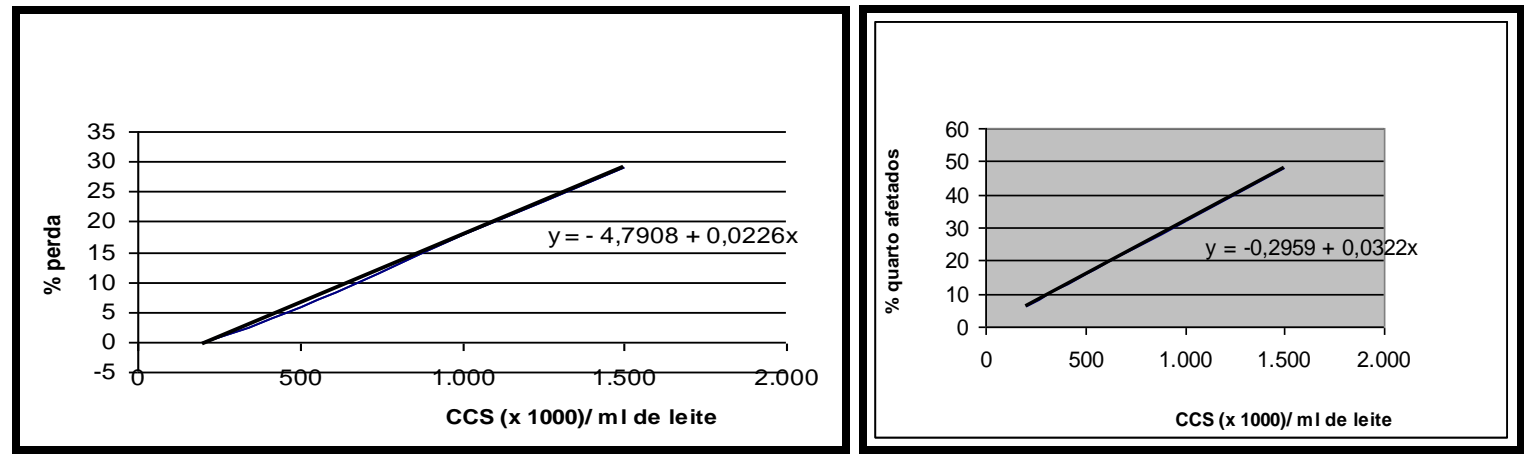

Figura 10. Tela contendo planilhas e gráficos

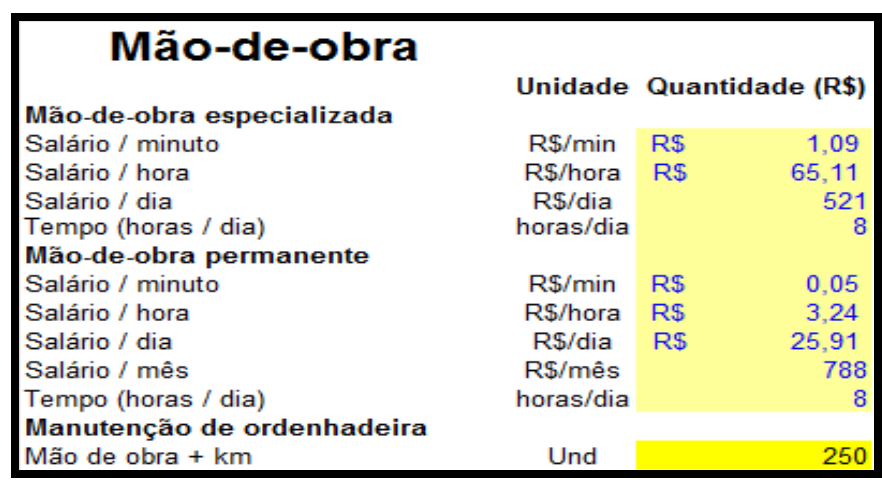

Figura 11. Tela contendo a planilha Mão de obra

\section{Conclusões}

O sistema computacional desenvolvido é uma importante ferramenta que auxilia o técnico e o pecuarista na estimativa do impacto da mamite, fornecendo informações importantes para a tomada de decisões pelos pecuaristas; permite ao usuário diversas simulações englobando diversos parâmetros e índices envolvidos em um sistema de produção de leite. Tais simulações podem ter o intuito de traçar metas a serem alcançadas dentro do sistema de produção.

\section{Referências Bibliográficas}

Fetrow, J., Stewart, S., Eicker, S., Farnsworth, R., Bey, R. (2000). Mastitis: an economic consideration. In: Proceeding Annual Meeting National Mastitis Council, p.39, Verona, USA.

Müller, E. E. (2000). Profilaxia e controle da mamite. In: Workshop Sobre Produção e
Qualidade do Leite, 2000, Maringá. Anais... Maringá: 10-13.

Müller, E. E. (2002). Qualidade do leite, células somáticas e prevenção da mamite. In: SulLeite: Simpósio Sobre Sustentabilidade da Pecuária Leiteira na Região Sul do Brasil, 2002, Maringá. Anais... Maringá, 206-217.

Lopes, M. A. (1997). Informática aplicada à bovinocultura. Jaboticabal: FUNEP, 82p.

Lopes, M. A. (2002). Informática aplicada à bovinocultura leiteira. UFLA: Lavras. 130p. (Apostila do curso de especialização por tutoria à distância bovinocultura leiteira: manejo, mercado e tecnologias).

Lopes, M. A. \& Carvalho, F. de M. (2000). Custo de produção do leite. Lavras: UFLA. p.42, Boletim Agropecuário, 33. 
National Mastitis Council. (1996). Current concepts of bovine mastitis. 4. ed. Madison, p.64,.

Ribeiro, M. E. R.; Petrini, L. A.; Barbosa, R. S.; Zanela, M. B.; Gomes, J. F.; Stumpf Junior, W. \& Schramm, R. (2006). Ocorrência de mastite causada por nocardia spp. em rebanhos de unidades de produção leiteira no sul do rio grande do sul. Revista Brasileira de Agrociência, 12, 471-473.

Santos, M.V. (2002). Efeito da mamite sobre a qualidade do leite e derivados lácteos. In: Congresso Panamericano de Qualidade do Leite e Controle da Mamite, 2., Ribeirão Preto. Anais... Ribeirão Preto, 179-188.
Recebido em Dezembro 21, 2015

Aceito em Janeiro 14, 2016

License information: This is an open-access article distributed under the terms of the Creative Commons Attribution License, which permits unrestricted use, distribution, and reproduction in any medium, provided the original work is properly cited. 